\title{
Hsa_circ_103973 Acts as a Sponge of miR-335 to Promote Cervical Cancer Progression
}

This article was published in the following Dove Press journal: OncoTargets and Therapy

\author{
Yingping Zhu' \\ Xuelu Jiang' \\ Shuo Zhang $\mathbb{D}^{2}$ \\ Lingcong Wang $\mathbb{1 D}^{3}$ \\ Qun Zhou' \\ Jun Jiang'
}

'Department of Obstetrics and Gynecology, The First Affiliated Hospital of Zhejiang University of Traditional Chinese Medicine, Hangzhou 310006, People's Republic of China; ${ }^{2}$ Department of Gastroenterology, The First Affiliated Hospital of Zhejiang University of

Traditional Chinese Medicine, Hangzhou 310006, People's Republic of China;

${ }^{3}$ Department of Critical Care Medicine, The First Affiliated Hospital of Zhejiang University of Traditional Chinese Medicine, Hangzhou 310006, People's Republic of China
Correspondence: Jun Jiang; Yingping Zhu Department of Obstetrics and Gynecology, The First Affiliated Hospital of Zhejiang University of Traditional Chinese Medicine, No. 54, Youdian Road, Shangcheng District, Hangzhou 310006,

People's Republic of China

Tel/Fax +86 57I-86620269

Email 3282241258@qq.com;

jiangjun@zcmu.edu.cn
Background: Cervical cancer (CC) ranks as the second most common malignancy in women, accounting for more two 2 million deaths every year in the world. Recently, circular RNAs (circRNAs) have been reported to regulate the progression of multiple human tumors; however, whether it involves in CC remains largely elusive.

Materials and Methods: Two GEO circRNA expression profiles (GSE102686, GSE113696) were downloaded to analyze the differentially expressed circRNAs using bioinformatics methods. Expression of circ_103973, miR-335 and PPP6C in CC tissues and cell lines were examined by qRT-PCR. Cell apoptosis was assessed with PI/Annexin$\mathrm{V}$ double staining followed by the analysis of flow cytometry. Cell proliferation was evaluated by MTT and colony formation assays. Interaction between circ_103973 and miR-335, as well as miR-335 and PPP6C, were verified by dual-luciferase reporter assay.

Results: Circ_103973 was found to be highly expressed in both GSE102686 and GSE113696 datasets as well as in CC tissue samples and cell lines. Higher levels of circ_103973 were correlated to a worse outcome of CC patients. Knockdown of circ_103973 significantly promoted CC cell apoptosis and inhibited CC cell proliferation in vitro. Mechanistically, we demonstrated that circ_103973 served as a sponge of miR-335, which directly targeted PPP6C in CC cells. miR-335 was found to be decreased in CC, while PPP6C was found to be increased in CC. Moreover, anti-miR-335 could reverse the inhibitory effects of circ_103973 knockdown on CC cell proliferation, and this phenomenon could be blocked by si-PPP6C.

Conclusion: Circ_103973 promoted CC cell proliferation in vitro by physically binding miR-335, which further targeted and regulated PPP6C.

Keywords: cervical cancer (CC), circ_103973, miR-335, PPP6C, proliferation, apoptosis

\section{Introduction}

Cervical cancer (CC) ranks as the second most common malignancy in women, accounting for approximately 500,000 new cases and more than 250,000 deaths every year in the world. ${ }^{1,2}$ Estimation from the American Cancer Society indicates that 13,170 new cases will be diagnosed in the United State in 2019, and more than a third of patients will die. ${ }^{3}$ Although the exact pathogenesis of CC remains largely unclear, increasing evidences have shown that the persistent infection of high-risk kind human papillomavirus (HR-HPV) might be one of the critical pathogenic factors in its initiation and progression. ${ }^{4}$ Recent years, with the development and extensive application of HPV vaccine, the incidence of $\mathrm{CC}$ has been reduced to a considerable degree, making the morbidity and mortality of $\mathrm{CC}$ under effective control. ${ }^{5}$ However, despite the tremendous advancement in medical technologies in the past decades, the molecular mechanisms of $\mathrm{CC}$ remain largely unknown. 
Therefore, it is urgent to understand more about the molecular mechanisms of CC and identify several novel critical agents for the diagnosis and therapy of CC.

Circular RNAs (circRNAs) are a newly identified type of non-coding RNAs in mammal cells with a circular structure. ${ }^{6}$ Unlike linear RNAs, circRNAs have no 5'-cap and 3'-poly A tail, which enable circRNAs resistant to the degradation of exonuclease and RNase $\mathrm{R}^{7}$ Due to this character, circRNAs have been considered to be more conservative and stable compared to multiple linear RNAs, such as micro RNAs (miRNAs) and long non-coding RNAs (lncRNAs). ${ }^{8,9}$ Moreover, circRNAs' expression usually exhibited cell-, tissue- and even time-specific manner. ${ }^{8}$ Thus, circRNAs were considered to be an important type of diagnostic and therapeutic agent of human tumors. ${ }^{10}$ During the past decades, circRNAs have demonstrated to play a critical role in the initiation and progression of multiple human cancers, including breast cancer, lung cancer and liver cancer. ${ }^{11}$ The implication of circRNAs in the pathogenesis of $\mathrm{CC}$ is supported by the microarray analysis showing the expression profiles of circRNAs are dysregulated in $\mathrm{CC}$ tissues compared to normal controls. $^{12,13}$ The dysregulation of circRNAs expression profile in $\mathrm{CC}$ tissues suggested that they might play a critical role in the tumorigenesis of CC. However, the roles of most of circRNAs in the pathogenesis of $\mathrm{CC}$ remain undetermined.

In the present study, we aim to identify specific circRNAs that correlated to the tumorigenesis of $\mathrm{CC}$, attempting to explore several novel diagnostic and therapeutic targets for $\mathrm{CC}$ patients. Bioinformatics analysis was performed in two GSE datasets (GSE102686 and GSE113696) to identify the dysregulated expressed circRNAs of CC tissue samples. As results showed that circ_103973 was identified to be upregulated in both GSE102686 and GSE113696 datasets. qRT-PCR detection of circ_103973 confirmed the upregulation of circ_103973 in CC tissue samples and cell lines. In the following functional assays, we found that circ_103973 might act as an oncogene of CC. In mechanism, circ_103973 was demonstrated to sever as a sponge of miR-335, which further targeted and regulated the expression of PPP6C. In conclusion, circ 103973 promoted CC cell proliferation in vitro by indirectly regulating the expression of PPP6C via sponging miR-335.

\section{Materials and Methods \\ CC Tissue Samples}

$\mathrm{CC}$ and adjacent normal tissue samples $(5 \mathrm{~cm}$ beyond the boundary of the cervical cancer tissues) were collected from 23 patients who underwent surgery at the First Affiliated Hospital of Zhejiang University of Traditional Chinese Medicine during 2015-2019. Patients were an average of 59 years old, with a range of 29-78 years. The inclusion criteria included patients with pathologically diagnosed cervical cancer; patients are willing to provide written informed consents. The exclusion criteria included patients who have received chemotherapy and radiotherapy; patients with cognitive and communication disorders; patients who do not cooperate with the examination; patients with other severe visceral diseases or complications. All patients signed an informed consent document for diagnosis and research on tissue specimens before being enrolled in the project. All subjects gave written informed consent in accordance with the Declaration of Helsinki principles. All tissue samples were dipped in liquid nitrogen immediately removed from patients and then stored at $-80^{\circ} \mathrm{C}$.

\section{Cell Lines}

The human cervical epithelial cell line (HCerEpiC), and four CC cell lines (HeLa, CaSki, C33A, SiHa) were provided by the Committee on Type Culture Collection of the Chinese Academy of Sciences (Shanghai, China). All types of cell lines were cultured in the Dulbecco's Modified Eagle's Medium (DMEM; Invitrogen, Waltham, MA) containing $10 \%$ fetal bovine serum, $100 \mathrm{U} / \mathrm{mL}$ of penicillin, $100 \mu \mathrm{g} / \mathrm{mL}$ of streptomycin (Sigma-Aldrich) under $5 \% \mathrm{CO}_{2}, 95 \% \mathrm{O}_{2}$ and $37^{\circ} \mathrm{C}$.

\section{Cell Transfection}

MiR-335 mimics, anti-miR-335 and corresponding scrambles were purchased from Genepharma Co., Ltd. (Shanghai, China). HeLa and C33A cells $\left(5 \times 10^{4}\right.$ cells/well $)$ were seeded in 6-well plates and transfected with scrambles, miR-335 mimics and anti-miR-335 using Lipofectamine $(2000$ Reagent (Invitrogen), respectively.

\section{RNA Transfection}

Specific shRNAs that target circ_103973 sh-circ_103973-1 (sh1), and sh-cicr_103973-2 (sh2), negative control (NC), miR-335 inhibitor (anti-miR-335), scramble, siRNAs of PPP6C (si-PPP6C), and si-NC were all designed and provided by GenePharma (Shanghai, China). For cell transfection, HeLa and C33A cells were plated into 6-well plates and cultured at $37^{\circ} \mathrm{C}$ for $24 \mathrm{hrs}$, and then cells were transfected with corresponding RNAs using Lipofectamine3000 
(Invitrogen, CA, USA) according to the manufacturer's instructions.

\section{Vector Construction and Transfection}

The PPP6C plasmid was purchased from Hanbio (Shanghai, China), and the circ_103973 plasmid was purchased from Genechem (Shanghai, China). Briefly, the extracted DNA was amplified using Taq PCR Master Mix Kit (Tiangen; Cat. no. 201443). The DNA productions were inserted into the pcDNA3.0 vector (Invitrogen) according to previous studies $[34,35]$. HeLa and C33A cells $\left(1 \times 10^{5}\right.$ cells/well $)$ were seeded in 6-well plates and transfected with the circ 103973 or PPP6C overexpression vectors and empty vector (control) using Lipofectamine ${ }^{\circledR} 2000$ Reagent (Invitrogen).

\section{RNA Extraction and Quantitative Real-Time PCR (RT-PCR) Assay}

Total RNAs were isolated CC tissue samples and cell lines using TRIzol reagent (Takara, Tokyo, Japan). RNA quality was examined by a NanoDrop 2000 Spectrophotometer (Thermo Scientific, Wilmington, DE, USA) according to the protocols of manufacturer. Reverse transcription of circ_103973 and PPP6C were carried out using PrimeScript RT reagent kit (Takara, Tokyo, Japan), while the reverse transcription reaction of miR-335 was conducted with a miRNA First Strand Synthesis Kit (Takara, Tokyo, Japan). The qRT-PCR process was conducted on an ABI 7500 fast real-time PCR system (Applied Biosystems, Darmstadt, Germany) with a SYBR Premix Ex Taq Kit (RR420A, Takara, Tokyo). GAPDH was regarded as the endogenous control for circ_103973 and PPP6C; U6 was used as control for miR-335 normalization. Fold changes in expression of RNAs were recorded through $2^{-\Delta \Delta C t}$. All sequences of primers are summarized as shown in Table 1.

Table I Primer Sequences Used In This Study

\begin{tabular}{|l|l|}
\hline Gene & Sequence or Target Sequence \\
\hline circ_103973-F & 5'-CTCCAGCTCAGACGCTTACC-3' \\
circ_103973-R & 5'-GGCTGGTATGGGTGAAAAGA-3' \\
GAPDH-F & 5'-CACCCACTCCTCCACCTTTG-3' \\
GAPDH-R & 5'-CCACCACCCTGTTGCTGTAG-3' \\
PPP6C-F & 5'-ACACAGGTGTATGGATTTTATGATG-3' \\
PPP6C-R & 5'-TGAGCATATCAAAAACTTTGGTACAG-3' \\
miR-335-F & 5'-TCAAGAGCAATAACGAAAAATGT-3' \\
miR-335-R & 5'-GCTG TCAACGATACGCTACGT-3' \\
U6-F & 5'-CTCGCTTCGGCAGCACA-3' \\
U6-R & 5'-AACGCTTCACGAATT TGCGT-3' \\
\hline
\end{tabular}

\section{Flow Cytometer Detection}

The treated HeLa and C33A cells were collected through centrifugation $(1200 \times \mathrm{g}$ for $5 \mathrm{mins})$, and the cells were counted using hemocytometer and adjusted cell concentration to $1 \times 10^{6} / \mathrm{mL}$. And then cells were fixed with $70 \%$ ethanol, treated with RNase A and stained with propidium iodide $(20 \mathrm{mg} / \mathrm{mL})$. Finally, the stained cells were all subjected for flow cytometry analysis (EPICS, XL-4, Beckman, CA, USA) following the instructions of manufacturers.

\section{Cell Proliferation Analysis}

Effects of circ_103973, miR-335 and PPP6C on cell proliferation of HeLa and C33A cells were evaluated by MTT and colony formation assays. For MTT assay, HeLa and C33A cells were seeded into 96-well plates at a density of 1000 cells/well and cultured overnight at $37^{\circ} \mathrm{C}$. Subsequently, 20 $\mu 1$ MTT solution was added into each well and incubated for $4 \mathrm{hrs}$. After discarding the supernatant, $150 \mu \mathrm{l}$ DMSO solution was added into each well and incubated for $15 \mathrm{mins}$ at room temperature. Finally, the absorbance of each well was determined at $490 \mathrm{~nm}$. For colony formation assay, treated $\mathrm{HeLa}$ and C33A cells were seeded into 6-well plates at a concentration of 3000 cells/well and maintained in a cell incubator with $5 \% \mathrm{CO}_{2}$ and $95 \% \mathrm{O}_{2}$ for 2 weeks. Subsequently, colonies were fixed using $4 \%$ paraformaldehyde followed by Giemsa staining for 30 mins. The visible colonies were counted manually.

\section{Dual Luciferase Reporter Assay}

For the establishment of recombinant luciferase reporter plasmids, the wild type (WT) and mutant (mut) fragments of circ_103973 containing putative miR-1205, miR-224, miR335 , miR-526b or miR-630 binding sites were amplified and inserted into the pGL3 (Promega, USA) vector. HeLa cells ( $2 \times$ $10^{4}$ ) were plated into 24-well plates and cultured overnight at $37^{\circ} \mathrm{C}$. Then, miR-1205, miR-224, miR-335, miR-526b or miR-630 and Luc-circ_103973-WT or Luc-circ_103973-Mut were co-transfected into HeLa cells using Lipofectamine 3000 reagent. After $48 \mathrm{hrs}$ of co-transfection, the luciferase activities of HeLa cells were measured by the Dual-Luciferase Reporter Assay System (Promega) following the manufacturer's instructions. The interaction between miR-335 and PPP6C in HeLa cells was also verified following the protocols above.

\section{RNA Pull-Down Assay}

HeLa cells stably transfected with biotinylated circ_103973 (Bio-circ_103973) or control (Bio-control) were harvested 
and lysed. HeLa cell lysates were incubated with C-1 magnetic beads (Life Technologies) at $4^{\circ} \mathrm{C}$ for $2 \mathrm{hrs}$ followed by the purification by RNeasy Mini Kit (QIAGEN, Duesseldorf, Germany). Finally, qRT-PCR was used to assess the expression of circ_103973 and miR-335.

\section{Statistical Analysis}

Statistical analysis was conducted on SPSS v20 software (IBM Corp., Armonk, NY, USA) using one-way analysis of variance. All error bars represent mean \pm SEM. P value less than 0.05 was considered as significant.

\section{Results}

\section{Circ_I03973 was Identified to Be Upregulated in CC}

To identify specific circRNAs that might be involved in the tumorigenesis of CC, two circRNA expression profiles (GSE102686 and GSE113696) were downloaded from the
GEO database (www.ncbi.nlm.nih.gov/geo). Volcano plots were formed using $\mathrm{R}$ software to show the differentially expressed circRNAs in GSE102686 and GSE113696 datasets, and circ_103973 was revealed to be dysregulated in both GSE102686 and GSE113696 datasets (Figure 1A). Results from GEO2R analysis indicated that circ_103973 was highly expressed in CC tissue samples compared to normal samples in GSE102686 and GSE113696 datasets (Figure 1B). To confirm the upregulation of circ_103973, we then detected its expression in both CC clinical tissue samples and cell lines using qRT-PCR. Results indicated that circ_103973 was significantly increased in CC clinical tissues compared to adjacent normal tissues (Figure 1C). Moreover, compared to the human cervical epithelial cell line (HCerEpiC), circ_103973 was also significantly upregulated in four CC cell lines (HeLa, CaSki, C33A, SiHa) (Figure 1D). In addition, we found that CC patients with high circ-103973 expression exhibited a worse prognosis than those patients with low circ_103973 expression (Figure 1E).
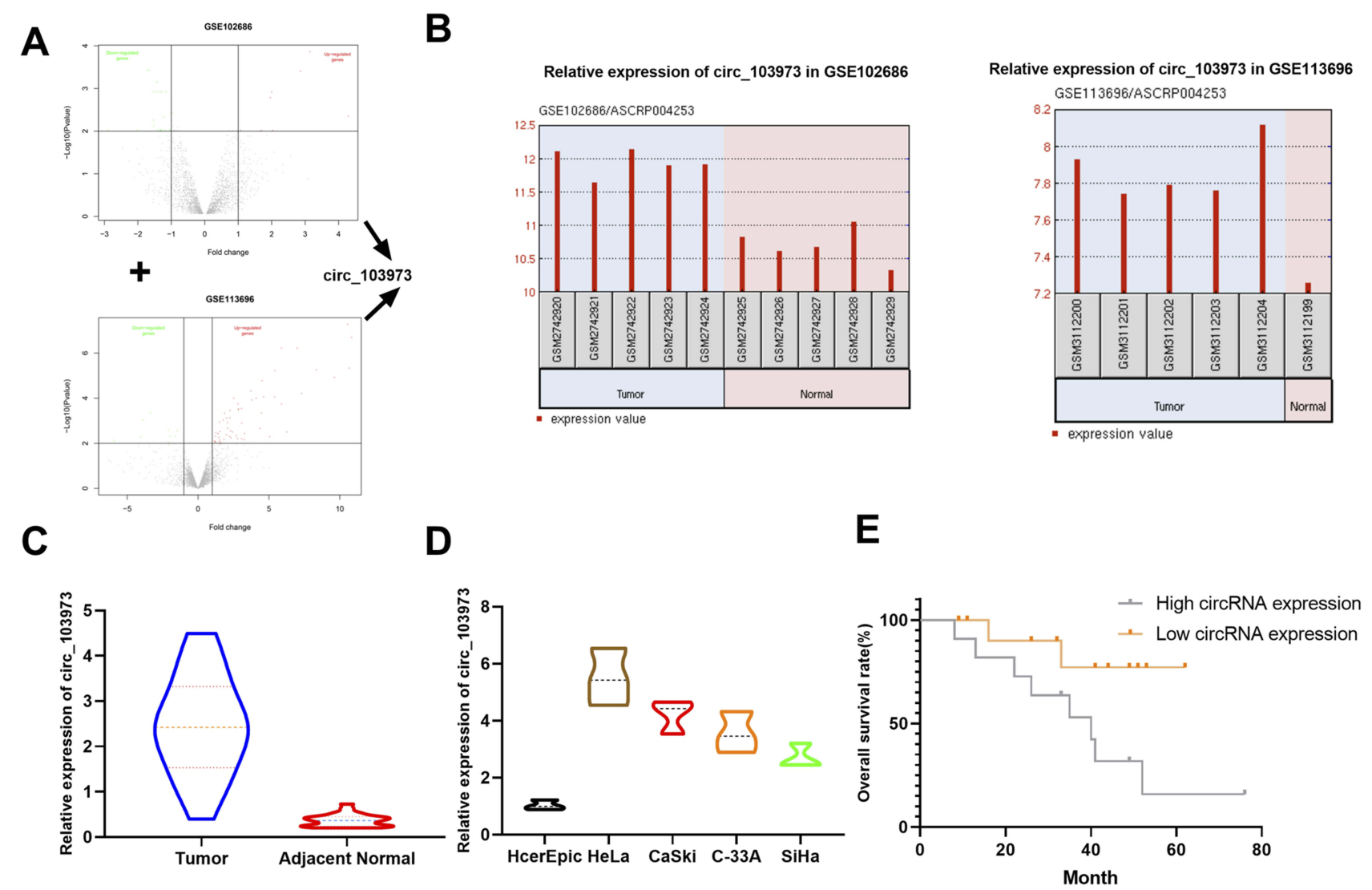

Figure I Circ_103973 was identified to be upregulated in CC. (A) Volcano plot of dysregulated circRNAs in GSEI02686 or GSEI I3696. (B) GEO2R analysis showing the expression of circ_103973 in CC and normal samples from GSEI02686 or GSEI I 3696 datasets. (C) Relative expression of circ_I03973 was measured in CC ( $\mathrm{n}=23$ ) and adjacent normal tissue samples $(n=23)$ by qRT-PCR. (D) Relative expression of circ_l 03973 was examined in human cervical epithelial cell line (HCerEpiC) and four CC cell lines (HeLa, CaSki, C33A, SiHa). (E) Kaplan-Meier analysis of overall survival rate of CC patients with low or high circ_I03973 expression. 


\section{Knockdown of circ_103973 Promoted} CC Cell Apoptosis and Inhibited CC Cell Proliferation in vitro

To investigate the biological functions of circ_103973 in CC, we silenced its expression in HeLa and C33A cells by specific shRNAs followed by the analysis of cell apoptosis and proliferation using flow cytometry, MTT and colony formation assays. Two specific shRNAs (sh1 and sh2) were designed to silence the expression of circ_103973 (Figure 2A). qRT-PCR showed a very high knockdown efficiency of circ_103973 shRNAs in HeLa and C33A cells (Figure 2B). For the apoptosis analysis using flow cytometry, circ_103973 shRNAs (sh1 and sh2) treated HeLa and C33A cells showed a significant upregulation of apoptosis rate than those cells treated with NC (Figure 2C). Results from MTT assay showed that circ 103973 shRNAs' treatment resulted in a remarkable inhibition of cell viability of HeLa and C33A cells (Figure 2D and $\mathrm{E})$. In the colony formation assay, we found that circ 103973 knockdown in HeLa and C33A cells could significantly reduce the number of colonies (Figure $2 \mathrm{~F}$ and $\mathrm{G}$ ). Moreover, in order to further explore the possible mechanism of circ_103973 knockdown affecting CC cell proliferation, flow cytometer was carried out to the impact of circ_103973 knockdown on cell cycle distribution of HeLa and C33A cells. As shown in Figure S1, the cell percentages in G1 and G2 phases were significantly increased, while the cell percentage in S phase was significantly reduced in circ_103973 siRNAs' transfection group compared to NC group, indicating that knockdown of circ_103973 promoted CC cell cycle arrest.

\section{Circ_103973 Targeted miR-335, Which Reversed the Inhibitory Effects of circ_103973 Knockdown on CC Cell Proliferation}

To investigate the molecular mechanism underlying circ_103973 in CC, bioinformatics analysis was performed
A

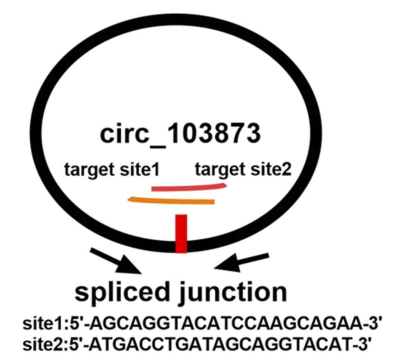

site2:5'-ATGACCTGATAGCAGGTACAT-3'

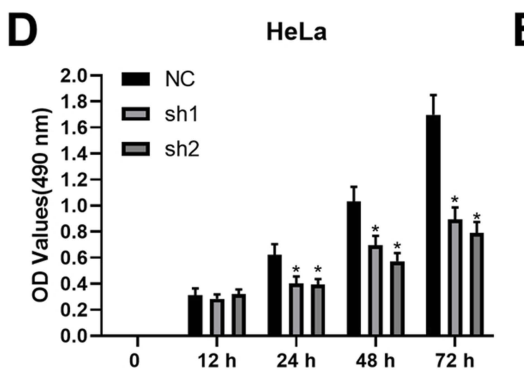

B

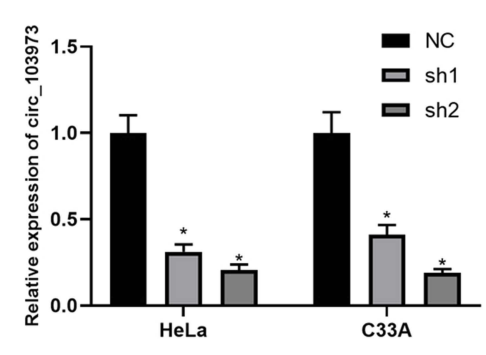

$\mathbf{E}$

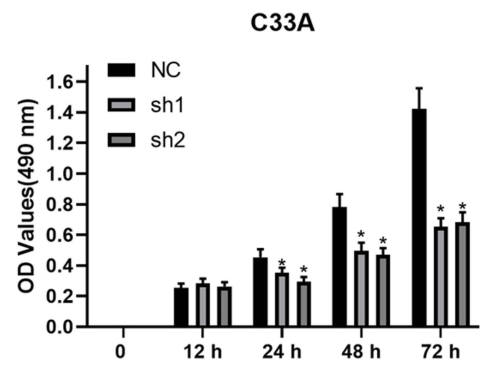

C

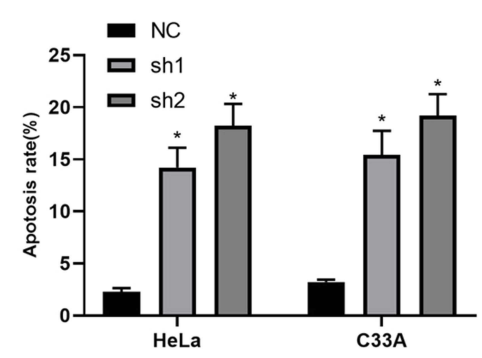

$\mathbf{F}$

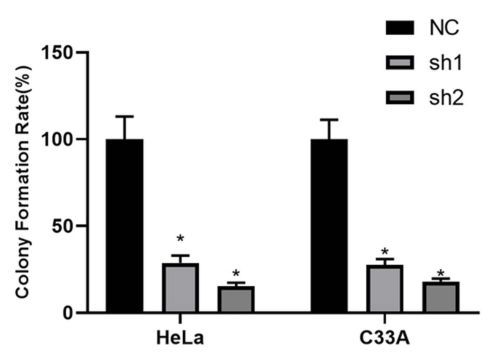

G

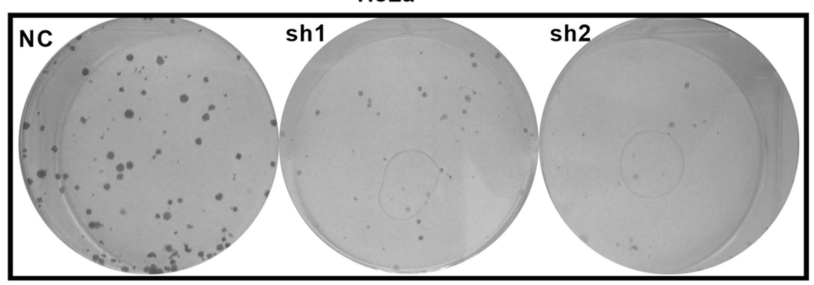

C33A

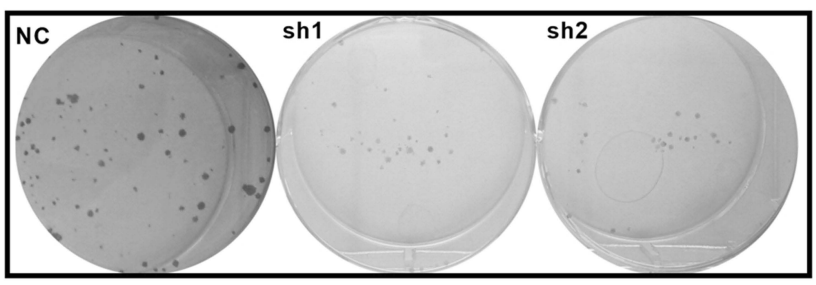

Figure 2 Knockdown of circ_103973 promoted CC cell apoptosis and inhibited CC cell proliferation in vitro. (A) Schematic diagram showing the target sites of circ_103973 shRNAs. (B) Knockdown efficiency of circ_103973 shRNAs (shRNAI and shRNA2) in HeLa and C33A cells was evaluated by qRT-PCR. (C) Apoptosis rate of HeLa and C33A cells transfected with circ_I03973 shRNAs (shRNAI and shRNA2) and NC. (D and E) Cell viability was examined in circ_I03973 shRNAs (shRNAI and shRNA2) and NC-treated HeLa and C33A cells by MTT assay. (F and $\mathbf{G}$ ) Colony formation assay was performed to assess the effects of circ_103973 shRNAs (shRNAI and shRNA2) on cell proliferation of HeLa and C33A cells. ${ }^{*} P<0.05$. 
to screen the target miRNAs of circ_103973 followed by the detection of target miRNAs using qRT-PCR in circ_103973overexpressed HeLa cells. Results indicated that the change of expression of five miRNAs (miR-1205, miR-224, miR-335, miR-526b and miR-630) was greater than 1-fold in circ_103973-overexpressed HeLa cells compared with empty plasmid-transfected HeLa cells (Figure 3A). In addition, we found that miR-335 showed a lowest level in circ_103973-overexpressed HeLa relative to miR-1205, miR-224, miR-526b and miR-630 levels. Therefore, mir-335 was selected as our research object. Next, dual-luciferase reporter assay showed that miR-224, miR-335, miR-526b and miR-630, but not miR-1205, could attenuate the luciferase activity of HeLa cells driven by Luc-circ_103973-WT (Figure 3B). The luciferase activity of HeLa cells driven by Luc-circ_103973 was not affected by miR-1205, miR-224,
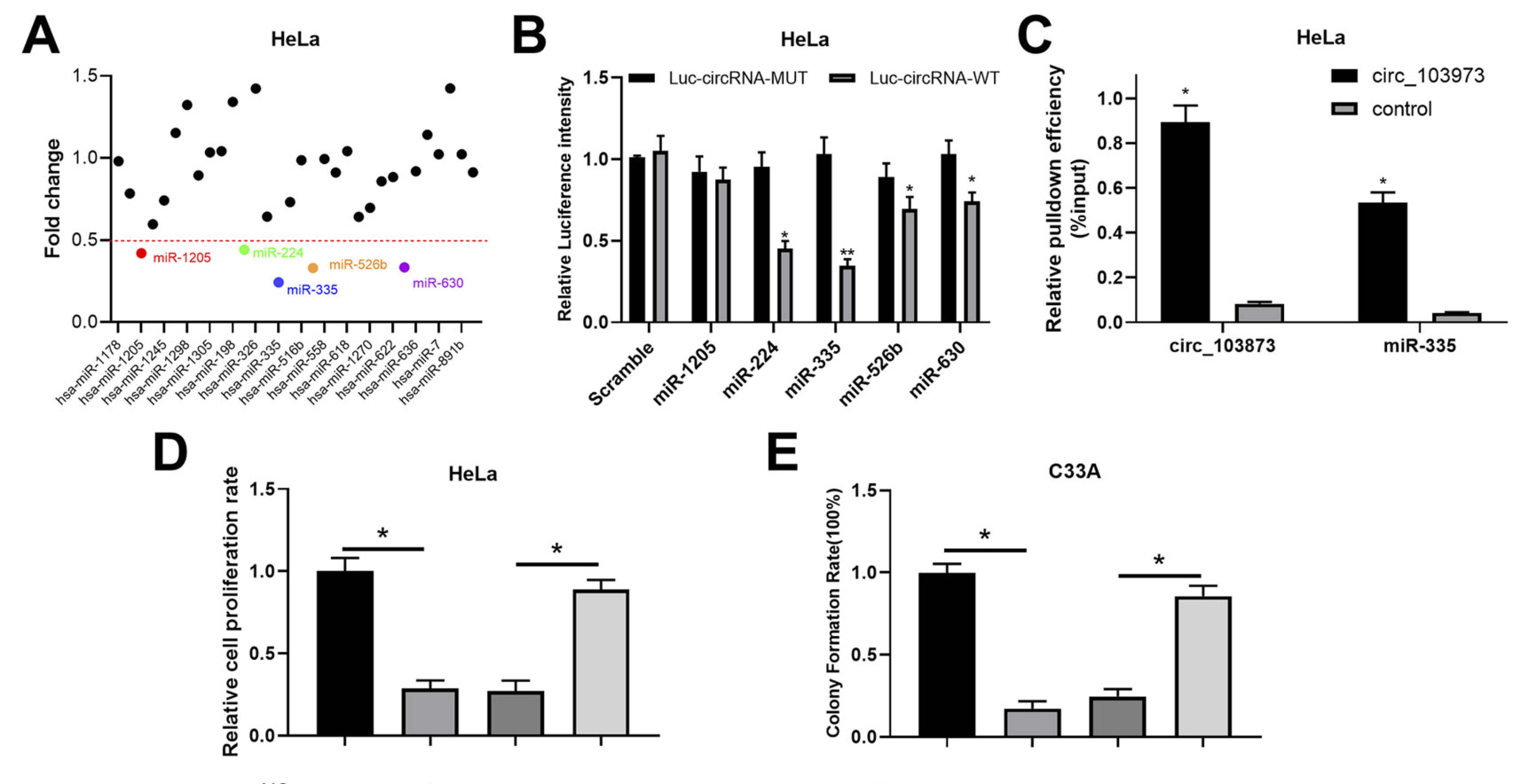

E

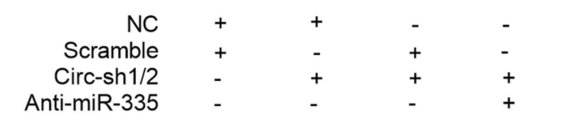

$F$
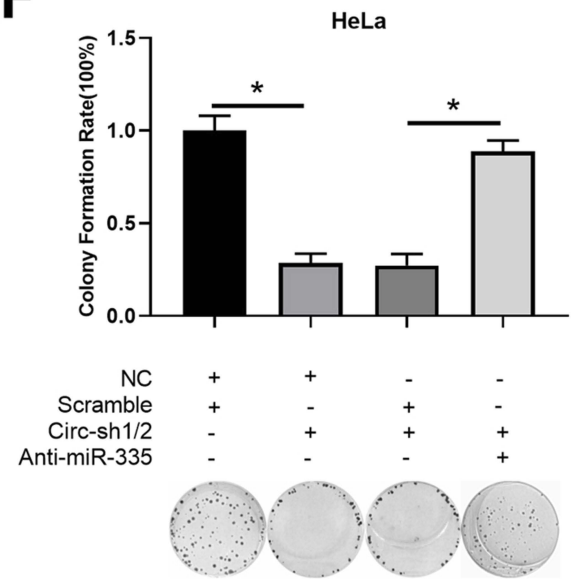

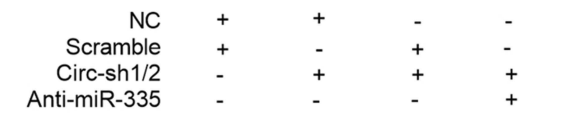

G
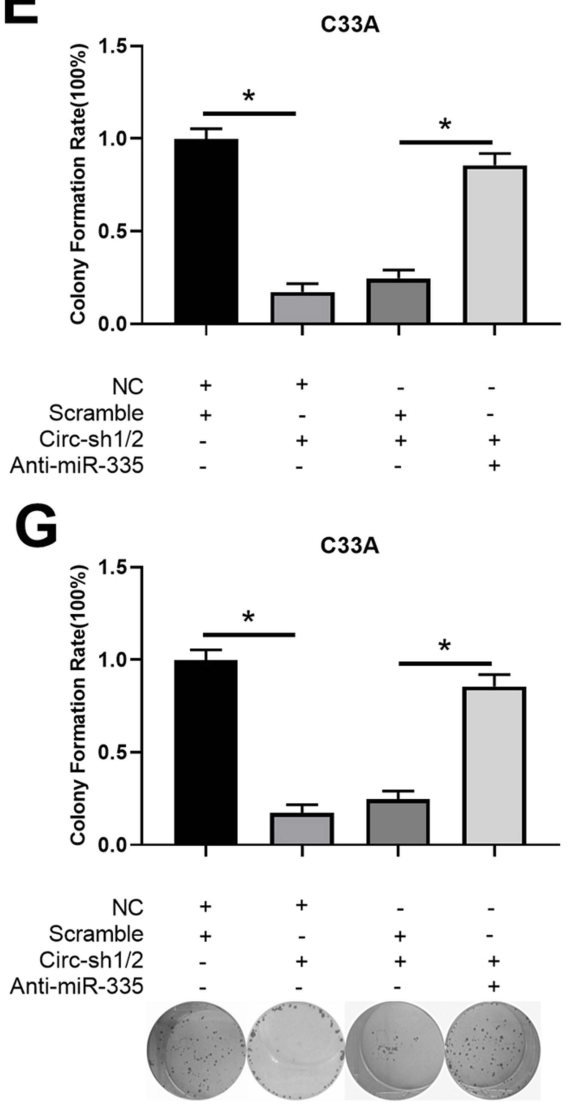

Figure 3 Circ 103973 targeted miR-335, which reversed the inhibitory effects of circ 103973 knockdown on CC cell proliferation. (A) Relative expression levels of multiple circ_103973 targeted miRNAs were measured in circ_103973-overexpressed HeLa cells. (B) Interaction between circ_103973 and miR-I205, miR-224, miR-335, miR-526b or miR-630 in HeLa cells was verified by dual-luciferase reporter assay. (C) RNA pull-down assay was performed in HeLa cells to further confirm the interaction between circ_103973 and miR-335. (D and E) Cell viability was measured by MTT assay in HeLa and C33A cells treated with NC+scramble, NC+circ_I03973 shRNAs, scramble+circ_103973 shRNAs, or circ_103973 shRNAs+anti-miR-335. (F and $\mathbf{G}$ ) Colony formation assay was carried out to detect the cell proliferation of HeLa and C33A cells treated with NC+scramble, NC+circ_103973 shRNAs, scramble+circ_103973 shRNAs, or circ_103973 shRNAs+anti-miR-335. $* P<0.05$, $* * P<0.01$. 
miR-335, miR-526b and miR-630. RNA pull-down assay was performed in HeLa cells to further confirm the interaction between circ_103973 and miR-335. Results indicated that the expression of miR-335 in circ_103973 group was significantly higher than that of control group (Figure 3C). Results from MTT assay showed that circ_103973 knockdown significantly inhibited cell viability of HeLa and C33A cells; however, this effect was abrogated by co-transfection of antimiR-335 and circ_103973 shRNAs (Figure 3D and E). Similarly, the reduced colony number of HeLa and C33A cells induced by circ_103973 was also reversed by cotransfection of anti-miR-335 and circ_103973 shRNAs (Figure 3F and $\mathrm{G}$ ).

\section{PPP6C was a Target Gene of miR-335 in CC Cell}

According to the miRBase prediction, miR-335 could target PPP6C 3'-UTR with a high score; dual-luciferase reporter assay was subsequently used to confirm the relationship between miR-335 and PPP6C in CC cells. The intensity of luciferase in CC cells driven by PPP6C-WT was attenuated by the transfection of miR-335, but not miR-NC; while the luciferase activity in those cells driven by PPP6C-MUT was not influenced by miR-335 or miR-NC (Figure 4A). After transfection of anti-miR-335, the expression of miR-335 was significantly downregulated, while the expression of PPP6C was remarkably upregulated in HeLa cells; after transfection of miR-335 mimics, miR-335 expression was significantly increased, while PPP6C expression was markedly decreased in HeLa cells (Figure 4B). To investigate the roles of PPP6C in circ_103973- and miR-335-mediated effects on TC, we silenced PPP6C by siRNAs and overexpressed PPP6C by overexpression vector, and the knockdown and overexpression efficiencies of si-PPP6C and PPP6C overexpression vector were examined via qRT-PCR assay (Figure 4C). The results of MTT assay revealed that miR-335 mimics transfection could significantly abolish the promoting effects of circ_103973 overexpression on HeLa and C33A cell proliferation, while these effects could be then reversed by
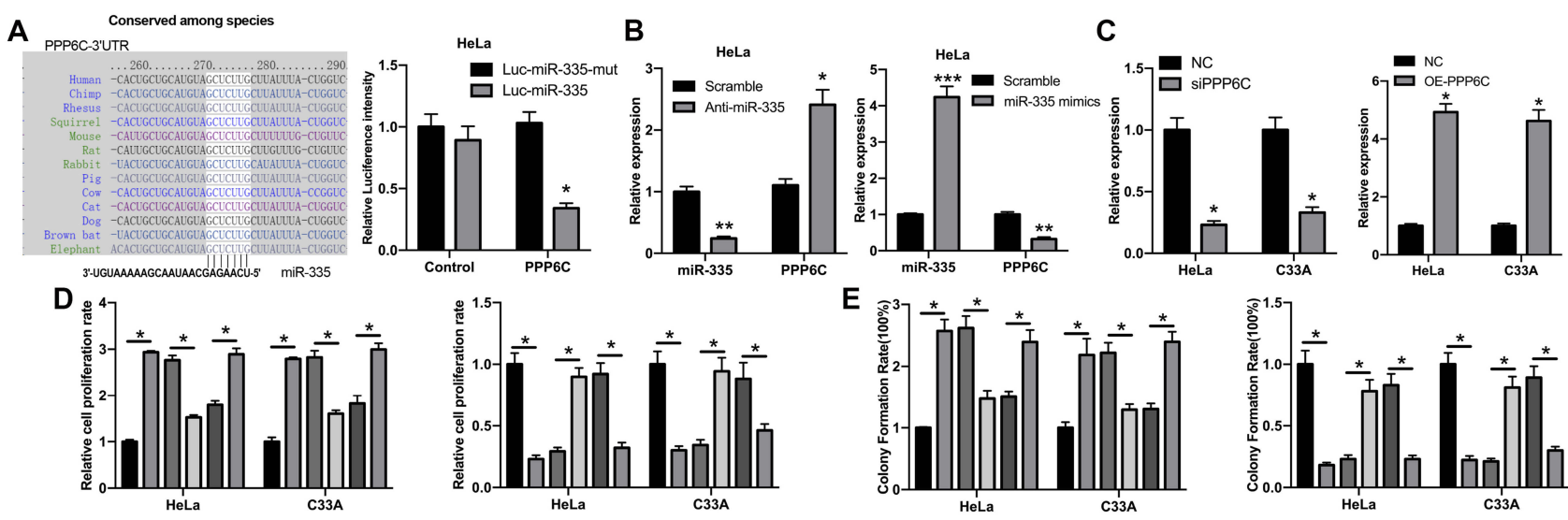

$E^{\prime}$
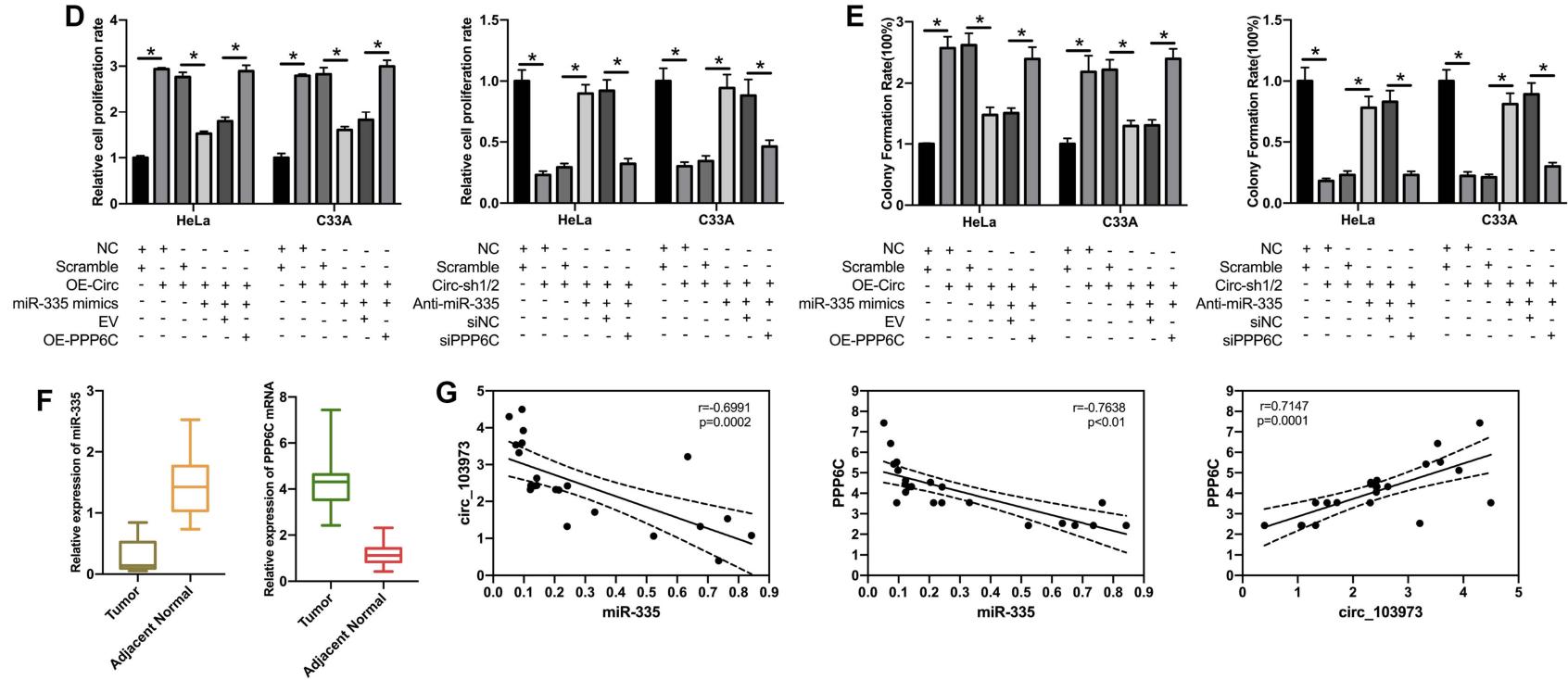

Figure 4 PPP6C was a target gene of miR-335 in CC cell. (A) The binding sites of miR-335 in the $3^{\prime}$-UTR of PPP6C were predicted by bioinformatics, and the interaction between PPP6C and miR-335 was determined by dual-luciferase reporter assay. (B) The levels of miR-335 and PPP8C were measured by qRT-PCR assay in HeLa cells transfected with anti-miR-335 or miR-335 mimics. (C) The knockdown and overexpression efficiencies of PPP6C were assessed through qRT-PCR assay in HeLa and C33A cells. (D) MTT assay was carried out to examine the effects of circ_103973, miR-335 and PPP6C on the proliferation of HeLa and C33A cells. (E) Colony formation assay was used to analyze the influences of circ_103973, miR-335 and PPP6C on the proliferation of HeLa and C33A cells. (F) Relative expression levels of miR-335 and PPP6C were detected by qRT-PCR assay in CC and adjacent normal tissue samples. (G) Correlations between miR-335 and circ I03973, miR-335 and PPP6C, PPP6C and circ_103973. $* P<0.05$, **P $<0.01$, *** $P<0.001$. 
the co-transfection of PPP6C in HeLa and C33A cells (Figure 4D, left). Anti-miR-335 treatment could significantly abolish the inhibitory effects of circ_103973 shRNAs on cell proliferation, while these effects could be reversed by the cotransfection of si-PPP6C in HeLa and C33A cells (Figure 4D, right). Similarly, the results of colony formation assay showed similar results with the results of MTT assay (Figure 4E). Data from qRT-PCR indicated that miR-335 was reduced, while PPP6C was increased in the $\mathrm{CC}$ tissue specimens compared to normal one (Figure 4F). A negative correlation was observed in the expression of circ_103973 and miR-335, as well as PPP6C and miR-335, in CC, while a positive correlation was revealed between the expression of PPP6C and circ_103973 in CC (Figure 4G).

\section{Discussion}

Implication of HPV infection in the etiology of $\mathrm{CC}$ has been widely recognized by public; therefore, the invention of HPV vaccine made a remarkable contribution to the primary prevention of $\mathrm{CC}$ in the world; however, there are still big challenges in the clinical treatment of $\mathrm{CC} .^{14,15}$ Illuminating the pathogenesis of $\mathrm{CC}$ is urgent to explore several effective biomarkers of $\mathrm{CC}$ for the screen and treatment of CC patients.

CircRNA was first reported by Sanger HL et al as an abnormal RNA produced by transcription disorder in the 1970 s in RNA viruses. ${ }^{16}$ It did not arouse much attention from the scientific community. However, with the tremendous advancement of high-throughput sequencing and bioinformatics methods, circRNAs have revealed to be expressed extensively in mammalian cells. ${ }^{17}$ In recent years, circRNAs are being proven to be a critical regulator of multiple biological processes in mammal cells, such as proliferation, apoptosis and differentiation. ${ }^{18}$ Therefore, circRNAs were considered to be important endogenous biomarkers of human tumors. ${ }^{18}$ Indeed, an amount of evidences have shown that the expression profiles of circRNAs were dysregulated in various human cancers, such as lung cancer, liver cancer and breast cancer. ${ }^{19}$ Recently, the biological functions of circRNAs in CC have also been well studied. For instance, circ_8924 was reported by $\mathrm{Li} \mathrm{W}$ to promote the $\mathrm{CC}$ cell proliferation, migration and invasion by sponging miR-518e-5p family and regulating the expression of $\mathrm{CBX} 8 .^{20}$ Moreover, results from Xianchao Kong el al showed that circ101996 might act as an oncogene of CC through increasing the expression of TPX2 through miR-8075. ${ }^{21}$ In this study, a circRNA microarray analysis was conducted on two CC-associated circRNA GSE datasets (GSE102686 and GSE113696) downloaded from the GEO database (www.ncbi.nlm.nih.gov/geo) to screen specific circRNAs that might involve in the pathogenesis of CC. Among the different expressed circRNAs, circ_103973 was identified to be significantly increased. After verifying the upregulation of circ_103973 in CC tissues and cell lines, we silenced its expression through specific shRNAs to examine the effects of circ_103973 knockdown on CC cell apoptosis and proliferation in vitro. As results indicated, circ_103973 might act as an oncogene in CC.

According to the hypothesis of competing endogenous RNAs (ceRNA), lncRNAs and circRNAs could sponge miRNAs complying the principle of complementary sequence, resulting in the liberating of gene transcripts that trapped by miRNAs. ${ }^{22,23}$ Therefore, we conducted bioinformatics analysis to predict the target miRNAs of circ_103973 in the present study, and multiple miRNAs were identified. Of these candidates, miR-335 was selected for further study. MiR-335 was previously reported to be associated with the progression of multiple human tumors, such as lung cancer, colorectal cancer and gastric cancer. $^{24-26}$ However, up to now, only one paper detected the role of miR-335 family in the pathogenesis of $\mathrm{CC}$, which suggested that IncRNA DANCR could promote CC progression by increasing the expression of ROCK1 through sponging miR-335-5p. ${ }^{27}$ Similarly, we found that circ_103973 acted as a sponge of miR-335, and inhibition of miR-335 in CC cells could abolish the effects of circ_103973 knockdown on CC cell proliferation. Furthermore, PPP6C was identified as targeted gene of miR-335. PPP6C was reported to be associated with multiple human diseases; ${ }^{28,29}$ however, its implication in CC remains undetermined. In our study, PPP6C was found to be upregulated in $\mathrm{CC}$, and si-PPP6C could abolish the effects of miR-335 knockdown on circ_103973 silenced $\mathrm{CC}$ cells.

\section{Conclusion}

In summary, our study demonstrated that increased circ_103973 promoted CC cell proliferation and inhibited CC cell apoptosis by directly increasing the expression of PPP6C through sponging miR-335. The circ_103973/miR335/PPP6C axis provided a novel insight into the complex molecular mechanisms of CC and revealed that circ_103973 might be a potential diagnostic and therapeutic target of CC. However, additional experiments need to be carried out further to verify the influencing mechanism of circ_103973/ 
miR-335/PPP6C axis on CC cell proliferation, such as cell cycle detection, 3D spheroids analysis and examination of markers related to cell proliferation and cycle. In addition, many in vivo experiments confirm the roles and underlining mechanisms of circ_103973/mir-335/PPP6C axis on CC cell progress including $\mathrm{CC}$ xenograft formation.

\section{Ethical Approval and Patient Consent}

This study was approved by the ethics committee of the First Affiliated Hospital of Zhejiang University of Traditional Chinese Medicine. All patients signed the written informed consent for this study.

\section{Author Contributions}

Conception and intellectual input: ZYP; designed and performed the experiments: ZYP, JXL, ZS, ZQ, JJ and WLC; manuscript drafting: ZYP. All authors contributed to data analysis, drafting and revising the article, gave final approval of the version to be published, and agree to be accountable for all aspects of the work.

\section{Disclosure}

The authors report no conflicts of interest in this work.

\section{References}

1. Eiben GL, da Silva DM, Fausch SC, Le Poole IC, Nishimura MI, Kast WM. Cervical cancer vaccines: recent advances in HPV research. Viral Immunol. 2003;16(2):111-121. doi:10.1089/088282403322017866

2. Marth C, Landoni F, Mahner S, et al. Cervical cancer: ESMO clinical practice guidelines for diagnosis, treatment and follow-up. Ann Oncol. 2017;28(suppl_4):iv72-iv83. doi:10.1093/annonc/mdx220

3. DeSantis CE, Miller KD, Goding Sauer A, Jemal A, Siegel RL. Cancer statistics for African Americans, 2019. CA Cancer J Clin. 2019;69:211-233. doi:10.3322/caac.v69.3

4. Walboomers JM, Jacobs MV, Manos MM, et al. Human papillomavirus is a necessary cause of invasive cervical cancer worldwide. J Pathol. 1999;189(1):12-19. doi:10.1002/(SICI)1096-9896(199909) 189:1<12::AID-PATH431>3.0.CO;2-F

5. Yee GP, de Souza P, Khachigian LM. Current and potential treatments for cervical cancer. Curr Cancer Drug Targets. 2013;13(2):205-220. doi:10.2174/1568009611313020009

6. Bach DH, Lee SK, Sood AK. Circular RNAs in cancer. Mol Ther Nucleic Acids. 2019;16:118-129. doi:10.1016/j.omtn.2019.02.005

7. Lasda E, Parker R. Circular RNAs: diversity of form and function. RNA. 2014;20(12):1829-1842. doi:10.1261/rna.047126.114

8. Liu L, Wang J, Khanabdali R, Kalionis B, Tai X, Xia S. Circular RNAs: isolation, characterization and their potential role in diseases. RNA Biol. 2017;14(12):1715-1721. doi:10.1080/15476286.2017.1367886

9. Holdt LM, Kohlmaier A, Teupser D. Molecular roles and function of circular RNAs in eukaryotic cells. Cell Mol Life Sci. 2018;75 (6):1071-1098. doi:10.1007/s00018-017-2688-5
10. He J, Xie Q, Xu H, Li J, Li Y. Circular RNAs and cancer. Cancer Lett. 2017;396:138-144. doi:10.1016/j.canlet.2017.03.027

11. Zhao ZJ, Shen J. Circular RNA participates in the carcinogenesis and the malignant behavior of cancer. RNA Biol. 2017;14(5):514-521. doi:10.1080/15476286.2015.1122162

12. Wang H, Zhao Y, Chen M, Cui J. Identification of novel long non-coding and circular RNAs in human papillomavirus-mediated cervical cancer. Front Microbiol. 2017;8:1720. doi:10.3389/fmicb.2017.01720

13. Ma HB, Yao YN, Yu JJ, Chen XX, Li HF. Extensive profiling of circular RNAs and the potential regulatory role of circRNA-000284 in cell proliferation and invasion of cervical cancer via sponging miR-506. Am J Transl Res. 2018;10(2):592-604.

14. Chelimo C, Wouldes TA, Cameron LD, Elwood JM. Risk factors for and prevention of human papillomaviruses (HPV), genital warts and cervical cancer. J Infect. 2013;66(3):207-217. doi:10.1016/j.jinf.2012.10.024

15. Wentzensen N, Arbyn M. HPV-based cervical cancer screening -c facts, fiction, and misperceptions. Prev Med. 2017;98:33-35. doi:10.1016/j.ypmed.2016.12.040

16. Sanger HL, Klotz G, Riesner D, Gross HJ, Kleinschmidt AK. Viroids are single-stranded covalently closed circular RNA molecules existing as highly base-paired rod-like structures. Proc Natl Acad Sci U S A. 1976;73(11):3852-3853. doi:10.1073/pnas.73.11.3852

17. Qu S, Yang X, Li X, et al. Circular RNA: A new star of noncoding RNAs. Cancer Lett. 2015;365(2):141-148. doi:10.1016/j.canlet.2015.06.003

18. Zhang Y, Liang W, Zhang P, et al. Circular RNAs: emerging cancer biomarkers and targets. J Exp Clin Cancer Res. 2017;36(1):152. doi:10.1186/s13046-017-0624-z

19. Patop IL, Kadener S. circRNAs in cancer. Curr Opin Genet Dev. 2018;48:121-127. doi:10.1016/j.gde.2017.11.007

20. Liu J, Wang D, Long Z, Liu J, Li W. CircRNA8924 promotes cervical cancer cell proliferation, migration and invasion by competitively binding to miR-518d-5p/519-5p family and modulating the expression of CBX8. Cell Physiol Biochem. 2018;48(1):173-184. doi: $10.1159 / 000491716$

21. Song T, Xu A, Zhang Z, et al. CircRNA hsa_circRNA_101996 increases cervical cancer proliferation and invasion through activating TPX2 expression by restraining miR-8075. J Cell Physiol. 2019. doi:10.1002/jcp. 28128

22. Poliseno L, Salmena L, Zhang J, Carver B, Haveman WJ, Pandolfi PP. A coding-independent function of gene and pseudogene mRNAs regulates tumour biology. Nature. 2010;465(7301):1033-1038. doi:10.1038/ nature 09144

23. Salmena L, Poliseno L, Tay Y, Kats L, Pandolfi PP. A ceRNA hypothesis: the Rosetta Stone of a hidden RNA language? Cell. 2011;146(3):353-358. doi:10.1016/j.cell.2011.07.014

24. Yang B, Huang J, Liu H, Guo W, Li G. miR-335 directly, while miR-34a indirectly modulate survivin expression and regulate growth, apoptosis, and invasion of gastric cancer cells. Tumour Biol. 2016;37(2):1771-1779. doi:10.1007/s13277-015-3951-8

25. Liu J, Bian T, Feng J, et al. miR-335 inhibited cell proliferation of lung cancer cells by target Tra2beta. Cancer Sci. 2018;109 (2):289-296. doi:10.1111/cas.13452

26. Lu Y, Yang H, Yuan L, et al. Overexpression of miR-335 confers cell proliferation and tumour growth to colorectal carcinoma cells. Mol Cell Biochem. 2016;412(1-2):235-245. doi:10.1007/s11010-015-2630-9

27. LiangH, Zhang C, Guan H, Liu J, Cui Y. LncRNA DANCR promotes cervical cancer progression by upregulating ROCK1 via sponging miR-335-5p. J Cell Physiol. 2019;234(5):7266-7278. doi:10.1002/ jcp. 27484

28. Shima H, Watanabe T. [Ppp6c deficiency predisposes mouse skin tissue to carcinogenesis]. Seikagaku. 2015;87(5):510-516.

29. Li X, Cai W, Xi W, et al. MicroRNA-31 regulates immunosuppression in ang II (Angiotensin II)-induced hypertension by targeting PPP6C (Protein Phosphatase 6c). Hypertension. 2019;73(5):e14e24. doi:10.1161/HYPERTENSIONAHA.118.12319 


\section{Publish your work in this journal}

OncoTargets and Therapy is an international, peer-reviewed, open access journal focusing on the pathological basis of all cancers, potential targets for therapy and treatment protocols employed to improve the management of cancer patients. The journal also focuses on the impact of management programs and new therapeutic

Submit your manuscript here: https:/www.dovepress.com/oncotargets-and-therapy-journal agents and protocols on patient perspectives such as quality of life, adherence and satisfaction. The manuscript management system is completely online and includes a very quick and fair peer-review system, which is all easy to use. Visit http://www.dovepress.com/ testimonials.php to read real quotes from published authors. 